\title{
PRÁTICAS EDUCATIVAS PARENTAIS, GÊNERO E ORDEM DE NASCIMENTO DOS FILHOS: ATUALIZAÇÃO
}

\author{
PARENTAL EDUCATIONAL PRACTICES, CHILD GENDER AND BIRTH \\ ORDER: AN UPDATE
}

Isabela T. A. Sampaio*

\begin{abstract}
Sampaio ITA. Práticas educativas parentais, gênero e ordem de nascimento dos filhos: atualização. Rev Bras Crescimento Desenvolv Hum 2007; 17(2):144-452.

Resumo: As práticas educativas parentais dizem respeito a como os pais monitoram, controlam e socializam seus filhos. Neste artigo, objetiva-se atualizar a literatura na área das práticas educativas parentais considerando-se especialmente dois fatores: o gênero (menino ou menina) e a ordem de nascimento (filho único, primogênito, do meio ou caçula) dos filhos. Foram consultados artigos nacionais e internacionais, sendo esses últimos considerados a partir do ano de 2001. Com relação às práticas educativas parentais e o gênero do filho, observou-se que parece existir uma preferência dos pais com filhos do mesmo sexo que o seu, as mães preferindo as meninas e os pais, os meninos. A literatura também demonstra que há diferenças no modo como meninos e meninas reagem a estressores familiares, sendo que os meninos apresentam maiores níveis de externalização e as meninas, de internalização de comportamento. Os artigos referentes à ordem de nascimento, por sua vez, discutem principalmente como é a alocação de recursos da família de acordo com a posição de nascimento de cada filho. Observou-se que os primogênitos, principalmente do sexo masculino, são os que recebem maior investimento dos pais. A literatura, porém, é frágil e contraditória com relação aos dois aspectos, assim, ressaltase que há necessidade de mais pesquisas sobre esses temas, especialmente no Brasil.
\end{abstract}

Palavras-chave: Práticas educativas parentais. Gênero. Ordem de nascimento.

Por práticas educativas parentais entendese a tentativa dos pais de controlar e socializar seus filhos ${ }^{1}$, às estratégias utilizadas por eles para criá-los ${ }^{2}$, e às técnicas que têm o objetivo de suprimir comportamentos considerados inadequados e/ ou incentivar a ocorrência de comportamentos adequados $^{3}$. A literatura a esse respeito é vasta; entretanto, neste artigo, pretende-se esboçar especificamente o que há de mais recente na literatura nacional e internacional sobre as práticas educativas parentais em relação ao gênero e à ordem de nascimento dos filhos, através de artigos publicados em importantes bases de dados nacionais (Scielo, Bvs-Psi) e internacional (PsicInfo), no últi- mo caso considerando apenas as publicações a partir do ano de 2001.

$\mathrm{O}$ enfoque ao gênero e à ordem de nascimento dos filhos é dado porque se acredita que são eles os principais fatores que influenciam o comportamento parental e a competição entre irmãos ${ }^{4}$. Isso justifica a preocupação atual da Psicologia em fornecer explicações sobre as diferenças encontradas entre irmãos, desmistificando a idéia de que filhos dos mesmos pais tendem ou deveriam ser muito parecidos em termos de personalidade ${ }^{5}$. Além disso, foi notada uma escassez de publicações brasileiras nas bases de dados citadas a respeito, principalmente, da

Psicóloga pela UFPR. Mestranda em Psicologia Programa de Pós-Graduação em PsicologiaUniversidade Federal de Santa Catarina Endereço para correspondência: Rua do Viamão, 379 Guabirotuba, Curitiba PR. Cep: $81520-380$ Email: izabelatissot@gmail.com 
influência da ordem de nascimento nas relações entre pais e filhos.

\section{Práticas educativas parentais em relação ao gênero dos filhos}

A literatura a respeito das práticas educativas parentais com relação ao gênero dos filhos demonstra que são vários os fatores que interferem nas interações existentes entre pais e filhos, como a relação de apego ${ }^{6}$, a forma de comunicação ${ }^{7}$, a identidade e a tipificação sexual, os papéis de homens e mulheres socialmente esperados, além das expectativas parentais acerca do sexo de seu filho ${ }^{8}$. A partir de uma revisão da literatura sociológica a respeito das diferenças entre meninos e meninas, Glória ${ }^{9}$ salienta que, embora esse pareça ser o tema mais abordado dentre as Ciências Sociais, ainda faltam trabalhos que o relacionem às práticas educativas familiares. Concordando com a autora, Daniels et al. ${ }^{10}$ criticam uma revista inglesa denominada "Every Child Matters" ressaltando a falta de análise de gênero e sua relação com as práticas parentais como foco de discussão, especialmente porque dados relativos a esse tema contribuiriam para a organização dos serviços prestados às crianças. Por outro lado, quando se procura nas livrarias brasileiras, encontram-se livros acerca da diferença dos cuidados parentais em relação ao gênero dos filhos, como Criando Meninos, do autor australiano Steve Biddulph ${ }^{11}$, e Criando Meninas, da autora alemã Gisela Preuschoff ${ }^{12}$, os quais inclusive se acham entre os mais procurados em lojas e bibliotecas no momento.

No entanto, a idéia de que o gênero seja uma variável forte na determinação do investimento parental não é compartilhada por todos pesquisadores da área. Os autores Keller e Zach ${ }^{13}$, por exemplo, alegam que as pesquisas recentes vêm apontando que o sexo do filho não é uma variável forte na determinação do investimento parental, contrariamente à ordem de nascimento dos filhos, a qual parece surtir um efeito relevante sobre ele.

Existe uma certa contradição dos dados encontrados por pesquisadores brasileiros com relação à contribuição do gênero na configuração do estilo parental. Essa contradição pode ser ilustrada por duas pesquisas de validação de instru- mentos de avaliação de estilos parentas. Gomide e Guimarães ${ }^{14}$, por exemplo, compararam o valor do índice do estilo parental, segundo o modelo da primeira autora, de dois grupos, um de escolas públicas e outro de particulares, com relação ao gênero. Os resultados demonstraram que, em relação às praticas educativas maternas, verificouse que as meninas da escola particular perceberam suas mães com maior índice de punição inconsistente (punidas ou não pelo mesmo comportamento) do que os meninos, e que as meninas da escola pública perceberam suas mães mais negligentes do que os meninos. Com relação às práticas parentais paternas, obteve-se que as meninas consideraram seus pais com maior nível de punição inconsistente do que os meninos. Nos três casos, observou-se que as meninas avaliaram suas mães ou seus pais com maior rigor do que os meninos.

Por outro lado, utilizando a escala de responsividade baseada no modelo de Diana Baumrind e adaptada para o contexto brasileiro, Weber et al. ${ }^{15}$ realizaram um estudo para observar a freqüência dos estilos parentais e sua relação com o gênero. Os resultados demonstraram que tanto as mães quanto os pais são um pouco mais exigentes com as filhas do que com os filhos; embora não se tenha encontrado diferenças significativas quanto à responsividade entre meninos e meninas, ou seja, aos cuidados parentais a eles dispensados. De modo geral, as diferenças com relação ao gênero não foram significativas; porém, uma análise mais aprofundada demonstrou que, de todos os meninos, 70,2\% consideraram seus pais negligentes e $29,8 \%$ autoritativos. De todas as meninas, $47,9 \%$ classificaram seus pais como negligentes e 52,1\% como autoritativos. Ao contrário dos resultados de Gomide e Guimarães ${ }^{14}$, nesse estudo os meninos pareceram mais rigorosos na avaliação de seus pais.

Assim, embora de modo geral não tenham sido acusadas diferenças estatisticamente relevantes entre os estilos parentais avaliados por meninos e meninas, algumas diferenças foram encontradas, sendo inclusive contraditórias, como o rigor dos participantes ao avaliar seus pais. Esses dados de certa forma vão ao encontro da afirmação de Keller e Zach ${ }^{13}$, os quais sugerem que o gênero não seja uma variável forte na 
determinação dos estilos parentais. Há necessidade, portanto, que mais estudos sejam realizados para a compreensão dessa contradição.

De acordo com as concepções evolucionistas a respeito da diferenciação do gênero para a reprodução humana, supõe-se que a maior variabilidade da reprodução potencial de machos quando comparada às fêmeas seja uma conseqüência da seleção sexual e da reprodução humana. De acordo com essa hipótese, um pai investe mais nos filhos homens se o ambiente econômico mantém-se estável e previsível ao longo das gerações, contando com o sucesso reprodutivo de sua prole. Em ambientes com recursos escassos e imprevisíveis, um pai possivelmente investiria primariamente nas filhas mulheres, já que seu sucesso reprodutivo é menos variável do que o dos homens ${ }^{16}$.

No entanto, embora esse estudo ${ }^{14}$ sugira que o investimento parental possa variar com relação a meninos e meninas, dependendo do ambiente em que se dá a reprodução, outras pesquisas demonstram que, em certas culturas, o investimento dos pais é mais forte com relação ao menino ${ }^{5,9,13}$. Ainda, há concepções psicológicas que argumentam que os pais têm preferência por filhos do mesmo sexo que o seu; de acordo com essa idéia, as mães teoricamente preferem as filhas (meninas) e os pais, os filhos (meninos). Essa idéia é bem ilustrada pela pesquisa de Keller e Zach $^{13}$ que, a partir de observações de 58 pais e mães em interação com bebês de três meses de idade, constataram que: 1 - as mães passaram mais tempo sozinhas com suas filhas e os pais sozinhos com seus filhos; 2 - juntos, pais e mães permaneceram mais tempo com os filhos homens; 3 - as mães demoraram-se mais alimentando suas filhas do que seus filhos; 4- as mães passaram mais tempo com sua filhas e os pais com os filhos. A única exceção nesse estudo foi a constatação que os pais demoraram-se mais em contato visual recíproco com as filhas e não com os filhos.

Por outro lado, certas pesquisas detalham interações mais específicas dentro da família, principalmente da mãe com o filho homem. Um estudo desenvolvido por Oliveira et al. ${ }^{15}$, por exemplo, demonstrou que parece existir uma tendência de as mães dos meninos relatarem maiores níveis de intrusão na vida de seus filhos do que as mães das meninas, pois elas julgaram que todas as ações e pensamentos dos filhos deveriam ser controlados por elas. Ehrensaft et al. ${ }^{18}$ também constataram que as mães de meninos de alto risco social foram as que apresentaram maior grau de transtorno de conduta e práticas educativas inadequadas, como baixa capacidade na implementação de conseqüências consistentes e previsíveis diante do mau comportamento do filho, e maiores níveis de rejeição e interações conflituosas. A baixa monitoria da mãe referiuse, especialmente, ao desconhecimento de onde o filho se encontrava, quais suas companhias e quais atividades realizava. Veenstra et al. ${ }^{19}$ acrescentam que a associação entre condições econômicas desfavoráveis e frustração com o comportamento anti-social é mais forte nos meninos.

Um outro fator socialmente relevante ao se estudar o tema diz respeito ao investimento de pais e mães com relação à escolaridade dos filhos. Glória ${ }^{9}$, por exemplo, ressalta que as diferenças de atitudes parentais na expectativa de maior ou menor escolaridade dos filhos homens ou mulheres são mais acentuadas nos meios menos instruídos e nas camadas menos favorecidas. No entanto, em camadas mais favorecidas, parece não haver diferenças quanto ao sucesso aspirado pelos pais aos filhos homens ou mulheres. O que ainda se questiona, porém, é o fato de que muitas mulheres diplomam-se em disciplinas consideradas menos exigentes e menos remuneradas do que os homens.

Para Glória9 ${ }^{9}$ embora o pai invista tanto na menina quanto no menino, ele parece cooperar mais com o filho homem, enquanto que as mães comunicam-se um pouco menos com o menino do que com a menina. A atividade da mãe parece não exercer influência específica no que se refere aos meninos, mas elas tornam-se mais ambiciosas quanto à escolaridade das filhas quando elas mesmas são profissionais ativas. A autora salienta que, a partir das pesquisas analisadas, inclusive no Brasil, o nível de escolaridade exigida pelos pais é maior para meninas do que para meninos; em contrapartida, é esperado que o filho homem obtenha maior sucesso profissional e financeiro. Percebe-se que existe uma contradição no tocante 
ao empenho esperado nos estudos e o sucesso profissional dos filhos por parte dos pais.

A literatura encontrada também sugere que as práticas educativas adotadas pelos pais de acordo com o sexo da criança influenciam o modo como os filhos reagem a certos estressores. Roelofs et al. ${ }^{6}$, por exemplo, investigaram a relação entre fatores negativos presentes na família (superproteção, rejeição e ansiedade) e sintomas de internalização (sentimentos de ansiedade e depressão) e externalização (comportamento destrutivo, agressivo e anti-social) de uma amostra não-clínica de 237 crianças entre 9 e 12 anos. Os resultados apontaram que a presença de fatores negativos no comportamento paterno teve mais impacto sobre os meninos; enquanto que os mesmos fatores nas mães tiveram mais impacto sobre as meninas. Da mesma forma, as mães apresentaram mais rejeição e superproteção com relação aos filhos do que os pais. Para os meninos, apenas o apego inseguro com relação aos pais (homens) explicou parte significativa da variação nos sintomas de ansiedade e depressão. Para as meninas, contudo, o estilo do apego teve menos proeminência nos sintomas de externalização e internalização, enquanto as práticas educativas parentais tiveram maior contribuição nesse aspecto.

Guttmannova $^{20}$ (2005), por sua vez, verificou que os meninos de 6 anos apresentaram maiores níveis de internalização e externalização de comportamentos problemáticos do que as meninas. Aprofundando o tema, a autora complementa que os fatores de risco mais proeminentes para os meninos dessa idade, de etnia branca, foram: viver na pobreza persistentemente, receber pouco apoio emocional dos pais e ser filho de mãe adolescente. Para as meninas, também de etnia branca, o fator de risco de mais efeito foi a falta de estimulação cognitiva por parte dos pais.

Ainda avaliando a internalização ou externalização segundo o gênero, Gaylord et al. ${ }^{21}(2003)$ investigaram as estratégias de enfrentamento, idade e gênero como moderadores da associação entre estressores familiares, internalização, externalização e rejeição dos pares de 228 crianças entre 9 e 12 anos. Embora não tenha sido observada associação entre esses fatores com a idade e estratégias de enfren- tamento, foi percebido que as crianças que mais sofriam com estressores familiares foram as mais rejeitadas por colegas. O gênero não moderou a aceitação ou rejeição pelos pares, mas apresentou relação com a internalização. Especificamente, quanto mais crescia o número de estressores familiares, menor era a internalização entre as meninas, o que não ocorria entre os meninos. Para justificar essa diferença, os autores retomam outros pesquisadores que sugerem que as meninas têm maior avanço social na infância devido ao maior contato com modelos positivos do mesmo sexo. Além disso, especificamente com relação ao estudo descrito, os autores lembram que aproximadamente metade das crianças vivia em casas administradas por mulheres, corroborando o dado de que as meninas que vivem em famílias cuidadas por mulheres apresentam melhores resultados do que os meninos.

Adicionando outras variáveis ao estudo das práticas parentais e do comportamento infantil, Oldehinkel et al. ${ }^{22}$ (2006) estudaram a relação entre o temperamento (medo/receio e frustração) sintomas depressivos e práticas parentais de 2230 pré-adolescentes entre 10 e 12 anos do norte da Holanda. Os resultados apontaram que todos os fatores ligados às práticas parentais e ao temperamento dos filhos foram significativamente associados aos problemas depressivos nos préadolescentes. A frustração aumentou o efeito depressogênico da superproteção ou da falta de afeto dos pais. $\mathrm{O}$ medo/receio aumentou o efeito da rejeição dos pais com relação às meninas, mas não com relação aos meninos. Além disso, a associação entre frustração e depressão foi maior nos meninos.

No mesmo sentido, Hastings ${ }^{23}$ (2005) avaliou a relação entre gênero, inibição e socialização parental para o desenvolvimento do comportamento pró-social de 46 meninos e 42 meninas de idade pré-escolar avaliados durante dois anos. Não foi encontrada relação entre a inibição da criança e modo de se comportar do pai com o desenvolvimento do comportamento pró-social. No entanto, o mesmo não aconteceu com relação às mães. $\mathrm{O}$ comportamento materno foi preditivo do comportamento pró-social de maneira mais forte com as meninas mais inibidas. Para os auto- 
res, esses achados sustentam a hipótese de que comportamentos específicos dos pais dependem do temperamento e do gênero dos filhos.

A partir desses estudos, constata-se que existem alguns pontos em comum entre as diversas pesquisas referenciadas. A primeira conclusão a que se pode chegar é que, de modo geral, realmente existem diferenças no modo como pais e mães relacionam-se com seus filhos e filhas e que, portanto, meninos e meninas apresentam formas particulares de lidar com suas competências e dificuldades. O segundo fator relevante é a consistência dos dados que sugerem que os meninos apresentam mais problemas de internalização e, de maneira ainda mais proeminente, de externalização do que as meninas ao lidarem com fatores estressores, especialmente familiares. Isso está de acordo com a literatura a respeito do comportamento anti-social, segundo a qual esse tipo de comportamento aparece com mais freqüência e gravidade nos meninos. As meninas, por sua vez, parecem apresentar maiores índices de inibição/retraimento do que os meninos.

\section{Práticas educativas parentais em relação à ordem de nascimento dos filhos}

A ordem de nascimento dos filhos, ou seja, a posição ordinal do filho na seqüência de nascimentos dentro de uma família, foi considerada um fator de pouca importância no exame das interações familiares durante seus primeiros estudos $^{5}$. Foi a partir da publicação do livro Born to Rebel, do historiador Frank Sulloway ${ }^{4}$, que voltou à tona o debate sobre a influência dessa variável na relação entre irmãos, entre pais e filhos e nas características de personalidade. Ao contrário dos livros escritos especialmente com referência à diferença de gênero, como citado anteriormente, não é encontrado material publicado de fácil acesso a respeito dos efeitos da ordem de nascimento dos filhos sobre o papel parental. Vale ressaltar que as pesquisas aqui citadas são, na sua maioria, internacionais, enquanto só foram encontradas duas pesquisas nacionais sobre esse tema.

Segundo Sulloway ${ }^{24}$, a ordem do nascimento revela informações acerca da paridade (igualdade) dos pais e do sistema de fratrias (número de irmãos) no qual a criança está inserida. Para o autor, a partir de uma perspectiva evolucionista, a competição entre irmãos pelo amor dos pais é considerada uma importante força que empurra a evolução humana, contribuindo para as diferenças da formação da personalidade. Assim, o investimento dos pais depende não só nas condições econômicas da família, mas também da ordem do nascimento de seus filhos. Um exemplo citado em Hertwig et al. ${ }^{25}$, acerca dos cuidados parentais de pássaros, indicou que os animais que vivem em um ambiente extremamente carente de recursos devem investir de forma pesada ou quase exclusiva em apenas um filhote, ignorando os demais. Somente quando os recursos aumentam é que a divisão mais igualitária torna-se possível.

Com relação às pesquisas brasileiras encontradas, uma delas ${ }^{26}$ relacionava a ordem de nascimento, gênero e nível sócio-econômico com ansiedade-estado (estado emocional transitório, marcado por sentimentos de tensão e apreensão, e por um aumento da atividade do sistema nervoso autônomo). Os resultados indicaram que os estudantes primogênitos de nível sócio-econômico médio-alto apresentaram menor nível de ansiedade-estado do que os primogênitos de nível baixo. As mulheres, como esperado, foram as que apresentaram maior nível de ansiedade, sendo que as primogênitas de nível sócio-econômico mais baixo obtiveram a maior pontuação nesse quesito. De posse desses resultados, o autor concluiu que a ordem de nascimento não é, por si só, um fator relevante no estudo da ansiedade; porém, quando associada ao nível sócio-econômico e sexo, ela parece surtir efeitos significativos.

A outra pesquisa brasileira encontrada não foi realizada por psicólogos, mas por médicos psiquiatras, que se dedicaram ao estudo dos filhos únicos. Os autore ${ }^{27}$ avaliaram o impacto de ser filho único sobre as características de relacionamento com pais e amigos, desempenho escolar, comportamento social e sexual a partir de uma amostra de 360 adolescentes entre 15 e 19 anos. Os resultados indicaram que o fato de ser filho único não diferiu com relação ao relacionamento estabelecido com os pais, amigos, namorados(as) 
e nem nas práticas esportivas. As diferenças apareceram nas atividades de lazer, as quais são realizadas mais individualmente pelos filhos únicos; na freqüência de intoxicação por drogas, que foi baixa entre os filhos únicos quando comparados aos demais (primogênitos e não-primogênitos); e com relação à opção sexual, em que os filhos únicos apresentaram uma taxa maior de respostas referentes às homo e bissexualidade. Embora essas duas pesquisas tratem da ordem de nascimento como uma variável importante, elas não a relacionam com as práticas parentais diretamente, o que sinaliza a falta de trabalhos brasileiros sobre o assunto.

A maioria das pesquisas encontradas recentemente (a partir do ano de 2001), portanto, é de caráter internacional e revela ser o primogênito, especialmente menino, o filho que recebe maior investimento dos pais. Os resultados da pesquisa de Keller e $\mathrm{Zach}^{13}$ indicaram que tanto as mães quanto os pais permaneceram mais tempo junto com o primogênito, sendo que esse número se elevou quando as mães e interagiam com as filhas e os pais com os filhos. No entanto, pais e mães, quando estavam juntos, permaneceram mais tempo com os primogênitos meninos.

Da mesma forma, Shebloski et al. ${ }^{28}$ avaliaram as ligações recíprocas entre tratamento parental diferenciado, parcialidade percebida e auto-valorização de 384 díades de irmãos adolescentes. Os resultados indicaram que a ordem de nascimento foi significativamente associada com a auto-valorização e percepção de tratamento diferenciado de mães e pais. Numa primeira análise, os primogênitos e aqueles que eram nascidos antes que o irmão (e não eram primogênitos) não demonstraram diferenças de auto-valorização na percepção da parcialidade parental quando comparados ao grupo de irmãos mais novos, os quais indicaram ter menores índices de autovalorização. No entanto, quando o primeiro grupo foi dividido em primogênitos e não-primogênitos, os segundos apresentaram menor auto-valorização do que os primeiros. Nesse caso, porém, a auto-valorização dos primogênitos não foi relacionada à percepção do tratamento parental.

Sobre a primogenitura, Laurent e Sebastian $^{29}$ perceberam que, ao estudar 1129 crianças autoras de atos infracionais na França, os primogênitos foram aqueles que apresentaram menor número de transgressões leves e graves quando comparados aos "nascidos do meio", o que corrobora a literatura consultada pelos autores. Da mesma forma, os primogênitos indicaram ter sido mais supervisionados pelos pais do que os demais, o que permitiu aos autores concluírem que a ordem do nascimento tem papel moderador no comportamento delinqüente, e que esse efeito é em parte induzido pelo controle parental diferenciado.

Outra diferença encontrada por Shebloski et al. ${ }^{28}$ diz respeito à percepção do comportamento parental diferencial, em que os primogênitos foram aqueles que mais perceberam a diferença no tratamento dos pais com relação aos filhos, o que sugere que os adolescentes mais velhos têm maior propensão a observar e comparar o comportamento parental do que adolescentes mais novos. Da mesma forma, os autores encontraram que, quando o membro mais novo da díade considerava que seu par era o filho favorito, sua auto-valorização diminuía.

O fator "idade" também teve efeito na comparação da ordem de nascimento e tratamento dos pais, demonstrando que os primogênitos mais novos foram tratados de forma mais negativa quando comparados aos primogênitos mais velhos do que seus irmãos. Os autores ${ }^{26}$ ressaltam que, embora a percepção dos primogênitos pareça predizer o posterior tratamento parental diferencial, não se sabe se são os primeiros que utilizam estratégias específicas para influenciar os pais, ou se eles são mais receptivos com os filhos mais velhos.

Entretanto, a despeito dos estudos que referenciam possíveis "preferências" dos pais por algum filho específico, como sugerido por Shebloski et al. ${ }^{28}$, Hertwig et al. ${ }^{25}$ argumentam que existe uma pretensão dos pais em investir de forma igualitária entre os filhos, pois a distribuição igualitária do investimento parental reduz o risco de que a linhagem familiar se extinga. No entanto, os autores discutem que é justamente essa tentativa "igualitária", chamada pelos autores de equity heuristic, que gera a desigualdade do investimento entre os filhos em algumas situações. 
Para eles, a partir de uma contabilidade matemática acerca do investimento parental, pode-se perceber que a tentativa dos pais de serem iguais com os filhos não se mantém quando há três ou mais crianças na família.

Segundo os autores ${ }^{25}$, quando na família há apenas um filho, ele recebe todo o investimento dos pais. Quando há dois filhos, a soma total do investimento será a mesma entre eles, embora o momento que o investimento ocorra varie. Assim, o primogênito recebe mais dos pais quando tem menos idade, nos anos de vida que antecedem o nascimento do irmão; enquanto o segundo recebe mais quando o irmão já é mais velho e não demanda tanto dos pais, podendo inclusive não residir na mesma casa. A diferença (ou desigualdade) no investimento só aparece, portanto, quando há três filhos na família. O filho do meio, contudo, não dispõe em nenhum momento de uma oportunidade de ser o "único" a receber o investimento, já que vai sempre dividi-lo com o irmão mais velho ou mais novo.

Para Hertwig et al..$^{25}$, porém, não só o filho do meio sofre desvantagens. O primogênito pode ser prejudicado com relação à falta de liberdade em atividades mais permissivas por parte dos pais, como emprestar o carro ou chegar tarde em casa. E o caçula pode sofrer com a menor disponibilidade dos pais em cuidá-lo, especialmente no início da vida, pois ainda há os irmãos para serem cuidados também. No entanto, a complexidade dos fatores envolvidos na distribuição de recursos entre os filhos ultrapassa essa análise. Os próprios autores alertam que as circunstâncias moderadoras da distribuição igualitária dependem da duração do período de crescimento, a qual, quanto maior, menos prejudicial será ao filho do meio; ao intervalo entre nascimentos que, quanto maior, também menos prejudicial será ao filho do meio; e o tamanho da fratria, a qual, quanto maior, menor investimento receberá o filho do meio.

O conceito de "filho do meio", contudo, não se restringe ao segundo filho. Ele inclui todos aqueles que nasceram entre o primeiro e último irmãos, sendo mais freqüente quanto maior o tamanho da família. Nesse sentido, Hertwig et al. ${ }^{25}$ alertam que alguns de seus dados são inconsistentes com outros achados. Eles citam que um desses achados indicou que, em famílias com 3 a 5 filhos, os segundos-nascidos foram aqueles mais prejudicados em termos de tempo de cuidados, considerando o espaço de nascimento variando de 1 a 4 anos entre os irmãos. Isso quer dizer que o tamanho da família altera significativamente o investimento dos pais com relação aos filhos, em especial com os filhos do meio. Um outro dado revelou de que os últimos foram os que menos relataram pedir ajuda emocional ou financeira da família quando em necessidade, o que sugere um distanciamento desses dos seus próprios pais ou parentes.

Um outro tema bastante citado na literatura considerando a ordem de nascimento refere-se à relação entre essa variável e o coeficiente de inteligência (QI). Um debate entre pesquisadores da área foi publicado recentemente no periódico American Psychologist. Rodgers et al..$^{30}$, questionando os modelos que propõem que o tamanho da família e a ordem de nascimento influenciam o QI de crianças, no sentido que, quanto maior a família e mais distante a ordem de nascimento, menor o QI, avaliaram dados coletados a partir do National Longitudinal Survey of Youth, nos Estados Unidos, de crianças entre 8 e 14 anos. Os autores concluíram que, realmente, os pais de famílias maiores apresentaram QI mais baixo; todavia, contrariando outros achados, a crença de que crianças criadas em famílias grandes e mais distantes na ordem de nascimento apresentam menor QI não foi confirmada.

As conclusões de Rodgers et al..$^{30}$ geraram controvérsias entre os estudiosos da área, principalmente considerando-se as implicações metodológicas de sua pesquisa. Zajonc ${ }^{31}$, por exemplo, alega que o tamanho da família e os efeitos da ordem de nascimento não são variáveis que se transformam em causas. Elas são condições que sustentam, mediam ou previnem um conjunto de diversos resultados, sendo um deles o escore num teste de inteligência. Da mesma forma, Michalski e Shackelford ${ }^{32}$ alegam que a análise da ordem de nascimento no modelo intra-familiar não dá conta de outras variáveis relevantes, como a mudança na dinâmica familiar ao longo dos tempos, especialmente do ponto de vista econômico, ou da própria mudança de atitudes dos pais, 
que podem se tornar mais calmos e experientes ao terem outros filhos além do primeiro.

Os dados fornecidos pelas pesquisas a respeito da ordem de nascimento circulam principalmente os temas relativos ao favoritivismo parental entre irmãos a partir do olhar dos filhos, ao investimento material e cognitivo por parte dos pais e, de forma menos proeminente, a interação entre eles. Ressalta-se que foram encontrados poucos trabalhos brasileiros a respeito da ordem de nascimento, o que sinaliza maior necessidade de pesquisas na área.

\section{CONSIDERAÇÕES FINAIS}

O estudo das práticas educativas parentais, por abarcar uma infinidade de variáveis de difícil controle, necessita que seja entendida a partir de diversos recortes sem que se perca a dinâmica global do sistema familiar. Como afirma Montandon $^{33}$, ao estudarmos as práticas educativas parentais não podemos desconsiderar a reciprocidade dos fatores envolvidos, ou seja, a relação dos pais com as crianças e o ponto de vista de cada um deles. Nesse artigo, objetivouse demonstrar que duas variáveis em especial podem contribuir de maneira significativa para a organização familiar, o gênero e a ordem do nascimento e que, portanto, não podem ser desconsideradas. Ressalta-se a necessidade de maiores estudos sobre os temas, especialmente no Brasil, visto que ele parece estar fora da discussão internacional que cresce na tentativa de compreender as contradições e sanar as dificuldades de avaliação dos fatores presentes nas relações familiares.

\begin{abstract}
Parental educational practices regard how parents monitor, control and socialize their children. This article aims to update the literature about this issue, considering especially two factors: child gender (boy or girl) and birth order (only child, first, middle and last born child). Brazilian and international articles were considered, the latter from 2001 onwards. In relation to parental educational practices and child gender, it was observed that parents seem to prefer children of the same sex as theirs (fathers prefer boys, and mothers prefer girls). The literature also shows that there are differences in the way boys and girls react to family stressors: boys usually externalize and girls internalize behaviors. The articles referring to birth order, on the other hand, discuss mainly how the family resources allocation works according to the birth order of each child. It was noted that firstborns, especially boys, receive the greatest part of parental investment. Literature, however, is fragile and contradictory in relation to both issues; thus, more research is needed, especially in Brazil.
\end{abstract}

Keywords: Parenting. Educational practices. Gender. Birth order.

\section{REFERÊNCIAS}

1. Baumrind D. The influence of parenting style on adolescent competence and substance use.

Journal of Early Adolescence 1991; 11(1): 56-95.

2. Gomide PIC. Inventário de Estilos Parentais (IEP), modelo teórico, manual de aplicação, apuração e interpretação. Petrópolis: Vozes; 2006.

3. Alvarenga P. Práticas educativas parentais como forma de prevenção de problemas de comportamento. In: Guilhardi HJ. Sobre comportamento e cognição. Santo André: ESETec; 2001. p. 52-57.

4. Sulloway FJ. Born to rebel: birth order, family dynamics, and creative lives. New York:

Pantheon Books; 1996.
5. Bee H. A criança em desenvolvimento. $7^{\text {a }}$. ed. Porto Alegre: Artmed; 1996.

6. Roelofs J, Meesters C, ter Huurne M, Bamelis L, Muris P. On the links between attachment style, parental rearing behaviors, and internalizing and externalizing problems in non-clinical children. Journal of Child and Family Studies 2006; 15(3): 319-32.

7. Braz FS, Salomão NMR. A fala dirigida a meninos e meninas: um estudo sobre o input materno e suas variações. Psicologia Reflexão e Crítica. 2002; 15(2): 333-4.

8. Alencar EMS. A criança na família e na sociedade. Petrópolis: Vozes; 1982.

9. Glória DMA. Relação entre escolaridade e 
diferenças constitutivas das fratrias, 2005. Paidéia 2005; 15(30): 31-42.

10. Daniels B, Featherstone B, Hooper B, Scourfield J. Why gender matters for every child matters. British Journal of Social Work 2005; 35(8): 1343-55.

11. Biddulph S. Criando meninos. São Paulo: Fundamento; 2002.

12. Preuschoff G. Criando meninas. São Paulo: Fundamento; 2003.

13. Keller H, Zach U. Gender and birth order as determinants of parental behaviour. International Journal of Behavioral Development 2002; 20(2): 177-84.

14. Gomide PIC, Guimarães AM. Efeitos das práticas educativas sobre o comportamento anti-social em crianças e adolescentes. Relatório de pesquisa PIBIC/CNPq (não publicado) 2003. Universidade Federal do Paraná, Curitiba, Paraná, Brasil.

15. Weber LND, Prado PM, Viezzer AP, Brandenburg OJ. Identificação de estilos parentais: o ponto de vista dos pais e dos filhos. Psicologia: Reflexão e Crítica 2004; 17(3): 323-31.

16. Voland E. Evolutionary ecology of human reproduction. Annual Review of Anthropology 1998; 27: 347-74.

17. Oliveira EA, Frizzo GB, Marin AH. Atitudes maternas diferenciais para com meninos e meninas de quatro e cinco anos. Psicologia Reflexão e Crítica 2000; 13(3): 363-71.

18. Ehrensaft MK, Wasserman GA, Verdelli L, Greenwald S, Miller LS, Davies M. Maternal antisocial behavior, parenting practices, and behavior problems in boys at risk for antisocial behavior. Journal of Child and Family Studies 2003; 12(1): 27-40.

19. Veenstra R, Lindenberg S, Oldehinkel AJ, De Winter AF, Ormel J. Temperament, environment, and antisocial behavior in a population sample of preadolescent boys and girls. International Journal of Behavior Development 2006; 30(5): 32-42.

20. Guttmannova K. Development of externalizing and internalizing behavior problems during middle childhood: risk and protective factors. Dissertation Abstracts International 2000; 66, 2-B. (UMI No. AA13166291).

21. Gaylord NK, Kitzmann KM, Lockwell RL. Child characteristics as moderators of the association between family stress and children's internalizing, externalizing, and peer rejection. Journal of Child and Family Studies 2003; 12(2): 201-13.
22. Oldehinkel AJ, Veesntra R, Ormel J, de Winter AF, Verhulst FC. Temperament, parenting and depressive symptoms in a population sample of preadolescents, 2006. Journal of Child Psychology and Psychiatry 2006; 47(7): 68495.

23. Hastings P. (2005). Links among gender, inhibition, and parental socialization in the development of prosocial behavior. MerrillPalmer Quartely 2005; 51(4): 467-93.

24. Sulloway F. Birth order, sibling competition, and human behavior. In: Davies P, Holcomb $H$. The evolution of minds: psychological and philosophical perspectives. Boston, MA: Kluwer; 2000.

25. Hertwig R, Davis JN, Sulloway FJ. Parental investment: how an equity motive can produce inequality. Psychological Bulletin 2002; 128(5): 728-45.

26. La Rosa J. Ansiedade, sexo, nível sócioeconômico e ordem de nascimento. Psicologia: Reflexão e Crítica 1998; 11(1): 59-70.

27. Fuchs SC, Tavares MB, Fuchs FC, Diligenti F, Abreu JRP, Rohde LA. Características de comportamento do filho único vs filho primogênito e não-primogênito. Revista Brasileira de Psiquiatria 2004; 26(1): 17-23.

28. Shebloski B, Conger KJ, Widaman KF. Reciprocal links among differential parenting, perceived partiality, and self-worth a threewave longitudinal study. Journal of Family Psychology 2005; 19(4): 633-42.

29. Laurent B, Sebastian R. Birth order and youth delinquent behaviour: testing the differential parental control hypothesis in a French representative sample. Psychology, Crime \& Law 2005; 11(1): 73-85.

30. Rodgers JL, Cleveland HH, van den Oord E, Rowe DC. Resolving the debate over birth order, family size, and intelligence. American Psychologist 2000; 55(6): 599-612.

31. Zajonc RB. The family dynamics of intellectual development, 2001. American Psychologist; 56(6/7): 490-6.

32. Michalski RL, Shackelford TK. Methodology, birth order, intelligence, and personality. American Psychologist 2001; 56(6/7): 520-1.

33. Montandon C. As práticas educativas parentais e a experiência das crianças. Educação e Sociedade 2005; 26(91): 485-507.

Recebido em 28/03/2007 Aprovado em 24/04/2007 